\title{
Non-cystic fibrosis bronchiectasis in children: Aetiology, clinical profile and outcome in a single unit
}

\author{
*K W D A Anuradha 1 , P K G Gunathilaka 2 , V P Wickramasinghe ${ }^{3}$
}

Sri Lanka Journal of Child Health, 2019; 48(2): 111-116

\begin{abstract}
Background: Bronchiectasis is characterized by irreversible dilatation of the bronchial tree. Though cystic fibrosis $(\mathrm{CF})$ is the commonest cause in the western world, non-CF causes are the major contributors in South Asia and are a major contributor to chronic respiratory morbidity.
\end{abstract}

Objectives: To analyse a group of children with non-cystic fibrosis bronchiectasis diagnosed in a resource poor setting regarding aetiology, clinical profile and outcome.

Method: A chart review of 32 children presented /referred to a single unit over a period of 18 months were diagnosed by high resolution CT scan. Lung functions were assessed using spirometry.

Results: Thirty two children (girls 20) with non-CF bronchiectasis with a mean age of 7.7 years (SD $=3.42$ ) were studied. Bilateral lung involvement was seen in 10 patients. Restrictive lung disease was seen at spirometry in 11. An identifiable aetiology was found in 24 children out of which 16 were thought to be post infective. Postural drainage was satisfactory in 15 and 3 children were dependent on home oxygen.

Conclusions: An identifiable cause of non-CF bronchiectasis was found in $75 \%$ patients. The commonest cause identified was post infective.

DOI: http://dx.doi.org/10.4038/sljch.v48i2.8702

(Key words: Non cystic fibrosis, bronchiectasis, clinical profile)

${ }^{1}$ Professorial Paediatric Unit, Lady Ridgeway Hospital for Children, Colombo, Sri Lanka, ${ }^{2}$ Lady Ridgeway Hospital for Children, Colombo, Sri Lanka, ${ }^{3}$ Faculty of Medicine, University of Colombo, Sri Lanka

*Correspondence: anujaya2008@gmail.com

iD https://orcid.org/0000-0003-0685-785X (Received on 24 May 2018: Accepted after revision on 20 July 2018)

The authors declare that there are no conflicts of interest Personal funding was used for the project.

Open Access Article published under the Creative Commons Attribution CC-BY License

\section{Introduction}

Bronchiectasis is a chronic respiratory disease characterised by irreversible abnormal dilatation of the bronchial tree with anatomical distortion with different radiological patterns ${ }^{1}$. Non-cystic fibrosis (non-CF) bronchiectasis is commoner than $\mathrm{CF}$ bronchiectasis in both developing and developed countries with a significant contribution to respiratory morbidity ${ }^{2}$. However, only very limited scientific data are available on its clinical profile with attempts to analyse the aetiology, complications, management as well as the outcome ${ }^{3}$.

Bronchiectasis has no precise clinical case definition, its severity varies and the outcome is variable ranging from minimum respiratory morbidity to death ${ }^{4}$. A high degree of clinical suspicion is needed when evaluating children with recurrent respiratory tract infections or signs suggestive of chronic respiratory insufficiency ${ }^{5}$. Children suspected to have bronchiectasis should undergo high-resolution computer tomography (HRCT) scan without delay to confirm the diagnosis and to define the distribution and severity of airway involvement ${ }^{6}$. Lung function tests in older children provide a measure of functional impairment and small-airway involvement ${ }^{7}$.

Analysis of the clinical profile of 80 children (mean age 9.6 years) with non-CF bronchiectasis in India showed an identifiable aetiology in $64 \%{ }^{8}$. Common causes were post-infectious (19), primary ciliary dyskinesia (12), and allergic bronchopulmonary aspergillosis (6). Five children had required surgery, mainly lobectomy, for the removal of severely affected segments/lobes ${ }^{8}$. A systematic review of 989 children with non-CF bronchiectasis, comprising published articles from 1966 to 2014, concluded that the majority of children with non-CF bronchiectasis had an identifiable underlying aetiology ${ }^{9}$. Common causes were severe pneumonia, B cell abnormalities, recurrent aspiration or aspiration of a foreign body and ciliary dyskinesia9. There are no data available on Sri Lankan children with bronchiectasis.

\section{Objectives}

To describe the clinical profile, aetiology, complications, specific management and outcome in children with non-cystic fibrosis bronchiectasis 
attending a tertiary care hospital in Colombo, Sri Lanka.

\section{Method}

Thirty two children with non-CF bronchiectasis managed in the Professorial Paediatric Unit, Lady Ridgeway Hospital, from September 2016 to March 2018 were recruited for the analysis. The sample consisted of children who were diagnosed during the study period, diagnosed before the period of study and followed up at our unit, or referred for specialized care after making the diagnosis at another institute. Bronchiectasis was diagnosed by an experienced radiologist based on an HRCT scan (based on the typical radiological features of bronchial dilatation) in the background of a compatible clinical history. Absence of typical clinical features and having two negative sweat tests done a minimum of 6 months apart were taken to exclude cystic fibrosis. A comprehensive review of each child was done by taking a detailed history from the most reliable caregiver, perusing the clinical records including radiological imaging, lung function tests, sputum culture results, details on exacerbations and the adherence to the management offered. Techniques of postural drainage were checked on each patient. Details were taken on to a pre-prepared data collection sheet. Each patient had undergone two sweat tests, immunological studies, bronchoscopy, bronchoalveolar lavage, complete screening for tuberculosis and echocardiogram.

Post infective bronchiectasis was considered when there was a history of complicated pneumonia / bronchiolitis (viral or bacterial) or measles infection with pneumonia requiring prolong hospitalization and respiratory support (in most of the patients) prior to the onset of persistent respiratory symptoms leading to the diagnosis of bronchiectasis. However, they were categorized as post infective only after excluding other aetiologies in these patients. All patients, except children with congenital structural pulmonary or bronchial abnormalities leading to bronchiectasis, were investigated for congenital and acquired immunodeficiency disorders. The tests performed were retroviral studies, serum immunoglobulin levels, isohaemagglutinins, immune response to vaccine antigens, flow cytometry and nitro blue tetrazolium (NBT) test. All were evaluated for tuberculosis with a careful history, clinical examination, chest x-ray, Mantoux test, biological material from respiratory tract (sputum, early morning gastric aspirates or broncho-alveolar lavage samples) for acid fast bacilli, mycobacteria culture and nucleic acid amplification test (NAAT/ GeneXpert MTB/RIF ).

Bronchoscopy was performed as an initial investigation when the bronchiectasis and the preceding infections were unilateral and confined to a particular lobe in view of identifying foreign body aspirations. However, all patients had undergone bronchoscopy at some point of the follow up to exclude possible structural abnormalities of the bronchial tree and in view of obtaining broncho-alveolar lavage. Children with a history suggestive of recurrent aspirations underwent milk scan and upper gastrointestinal (GI) contrast study to identify possible gastrooesophageal reflux disease and/or tracheooesophageal fistula. Due to the limited resources available to identify allergic bronchopulmonary aspergillosis (ABPA), it was considered only in patients with no identifiable aetiology after initial evaluation and the International Society of Human and Animal Mycology (ISHAM) working group criteria were used for the diagnosis ${ }^{10}$.

Postural drainage practised by parents was assessed during the initial interview in the case of previously diagnosed patients and three months after training in newly diagnosed patients. Children were followed up regularly with documentation on growth, exacerbations, sputum culture results and annual pulmonary function tests. Medical management was optimized in each patient.

\section{Results}

Thirty two patients (girls 20) with non-CF bronchiectasis were studied. The mean age of the study population was 7.7 years (SD 3.42) and the mean age at diagnosis of bronchiectasis was 5.2 years (SD 2.5). The indication to perform an HRCT scan in the majority (19) was the combination of recurrent moist cough and respiratory tract infection with persistent crepitations on examination. Sixteen of them gave a history of a complicated pneumonia in early childhood. Review of chest x-rays taken within a two year period prior to making the diagnosis of bronchiectasis was done and eight patients showed radiological evidence of the condition. 
Table 1: Distribution of indications for HRCT scan in children with non-CF bronchiectasis $(n=32)$

\begin{tabular}{|l|c|c|}
\hline \multicolumn{1}{|c|}{ Indication } & No. of children \\
\cline { 2 - 3 } & Male & Female \\
\hline Persistence of recurrent respiratory symptoms with or without intermittent infections & \\
Presence of a history of * complicated pneumonia/bronchiolitis & 07 & 10 \\
No history of complicated pneumonia & 01 & 02 \\
\hline Haemoptysis & 0 & 02 \\
\hline First episode of poorly resolving pneumonia with chest X-ray evidence of bronchiectasis & 01 & 04 \\
\hline $\begin{array}{l}\text { Recurrent multi-systemic infections and an episodes of complicated pneumonia (CVID, CGD } \\
\text { and Hyper IgM syndrome) }\end{array}$ & 02 & 01 \\
\hline $\begin{array}{l}\text { Follow up evaluation after lobectomy for congenital structural malformations (sequestration } \\
\text { and CPAM) }\end{array}$ & 01 & 01 \\
\hline \multicolumn{1}{|c|}{ Total } & 12 & 20 \\
\hline
\end{tabular}

*Complicated Pneumonia ${ }^{11}$ - Pneumonia with one or more of the following: para pneumonic effusions/ empyema, pericardial effusions, lung abscess, necrotising pneumonia, atelectasis

CVID: common variable immune deficiency, CGD: chronic granulomatous disease, CPAM: congenital pulmonary airway malformations

Twenty children had a late diagnosis as they were managed as poorly controlled asthma with steroid and salbutamol inhalers. Mean duration between the documented concern of 'recurrent respiratory tract infections' and the diagnosis of bronchiectasis was 3.8 years (SD 1.5). Six patients were referred to the centre by a paediatrician or a general practitioner with the suspicion of bronchiectasis while others were diagnosed when admitted with an exacerbation of respiratory illness.

The commonest aetiology was post infectious where 16 had one or more episode of complicated pneumonia requiring respiratory support during early childhood with other common aetiologies being excluded. Other aetiological factors that were found in this group are listed in table 2.

Table 2: Distribution of the aetiology according to sex

\begin{tabular}{|l|c|c|c|}
\hline \multicolumn{1}{|c|}{ Aetiology } & \multicolumn{2}{c|}{ Number of patients } \\
\cline { 2 - 4 } & Male & Female & Total \\
\hline Possible post infectious & 05 & 11 & 16 \\
\hline Common variable immune deficiency & 01 & 0 & 01 \\
\hline Hyper IgM syndrome & 0 & 01 & 01 \\
\hline Chronic granulomatous disease & 01 & 0 & 01 \\
\hline Post tuberculosis & 01 & 0 & 01 \\
\hline Post bronchiolitis obliterans & 0 & 01 & 01 \\
\hline Severe gastro-oesophageal reflux disease & 0 & 01 & 01 \\
\hline Intra lobar sequestration & 0 & 01 & 01 \\
\hline Post lobectomy for congenital pulmonary airway malformation & 01 & 0 & 01 \\
\hline No identifiable aetiology Total & 03 & 05 & 08 \\
\hline \multicolumn{1}{|c|}{} & 12 & 20 & 32 \\
\hline
\end{tabular}

Eight children had no identifiable aetiology. Diagnostic facilities for primary ciliary dyskinesia (PCD) were not available but there were no patients with the characteristic clinical profile of PCD. Bilateral lung involvement with bronchiectasis at the diagnosis was seen in 10 patients.

Ten children showed body mass index (BMI) for age and sex less than -2SD. The mean BMI for age standard deviation score was -1.17. Average episodes of exacerbations per year were 6 and the commonest organism isolated in sputum at infective exacerbations was Pseudomonas aeruginosa (Table 3)
Table 3: Organisms isolated in sputum cultures at each infective exacerbation

\begin{tabular}{|l|c|}
\hline \multicolumn{1}{|c|}{ Organisms Isolated } & No. of patients \\
\hline Streptococcus pneumonia & 4 \\
\hline Staphylococcus aureus & 11 \\
\hline Pseudomonas aeruginosa & 16 \\
\hline Moraxella catarrhalis & 19 \\
\hline Haemophilus influenzae & 9 \\
\hline
\end{tabular}

Only 23 patients were able to undergo spirometry evaluation with an adequate effort when they were relatively symptom free. Age less than 5 years and severity of disease were the barriers. Best FEV1/FVC (Forced Expiratory Volume in 1 second/Forced Vital Capacity) ratio was $>80 \%$ in 17 and $<80 \%$ in 6 children (Table 4 ). 
FVC was not reduced (with a predicted value of $>80 \%$ ) in the majority (12) of children. FVC was $60-80 \%$ in 7 patients. There were only 3 patients with a $\mathrm{FVC}<60 \%$ and they were on home oxygen therapy. Out of the 17 patient who showed restrictive lung pattern, the predicted FEV1 was more than $80 \%$ in 13 indicating pure restriction to air flow. FEV1 was less than $80 \%$ in 10 children with bronchiectasis.

Table 4: Distribution of lung functions assessed by spirometer

\begin{tabular}{|c|c|c|c|}
\hline \multirow{5}{*}{ FEV1/FVC } & \multirow{3}{*}{$\begin{array}{l}>80 \% \text { of predicted } \\
17=72 \%\end{array}$} & FVC predicted value $>80 \%$ & 7- Normal \\
\hline & & $\mathrm{FVC}=60-80 \%$ & 7- Mild restriction \\
\hline & & $\mathrm{FVC}<60 \%$ & 3-Moderate restriction \\
\hline & \multirow{2}{*}{$\begin{array}{l}<80 \% \text { of predicted } \\
6=28 \%\end{array}$} & FVC predicted value $>80 \%$ & 5-Possible obstructive \\
\hline & & $\mathrm{FVC}<80 \%$ & 1.Mixed disease \\
\hline
\end{tabular}

FEV1: Forced expiratory volume in 1 second, FVC: Forced vital capacity

Satisfactory postural drainage was followed by parents/caregivers of 15 children and the rest had deficiencies in the technique that they adopted. Pneumococcal vaccination was given for 24 patients. Only 20 patients had complied with regular follow up.

Three patients had undergone lobectomy for congenital pulmonary airway malformations, intra lobar sequestrations and bronchiectasis confined to right middle and lower lobes. Out of the sample three children are on home oxygen therapy and were dependant on parents for activities of daily living.

Complications identified after the diagnosis and surgical interventions carried out are shown in Table 5.

Table 5: Complications identified after the diagnosis and surgical interventions

\begin{tabular}{|l|c|}
\hline \multicolumn{1}{|c|}{ Complications and surgical interventions } & Number of patients \\
\hline Chronic pseudomonas colonization & 05 \\
\hline Moderate to severe restrictive lung disease & 10 \\
\hline Massive haemoptysis & 02 \\
\hline Chronic hypoxaemia and oxygen dependency & 03 \\
\hline Pulmonary hypertension & 09 \\
\hline Surgical interventions (lobectomy/selective bronchial artery embolization) & 04 \\
\hline
\end{tabular}

\section{Discussion}

Non-CF bronchiectasis, though considered a declining condition in the developed world, is not rare among the paediatric population in developing countries. The group of children studied above with non-CF bronchiectasis showed many characteristics similar to the clinical profiles in the published literature. Identifiable aetiology was seen in $75 \%$ of children in this study group.

Analysis of 41 patients with bronchiectasis in Tunisia reported that the mean age at the time of diagnosis was 5 years and 9 months and that the mean time taken for diagnosis from onset of symptoms was 2.7 years $^{12}$. The mean age of diagnosis and time taken for diagnosis in our study were 5.2 years and 3.8 years respectively. Delay in diagnosis of bronchiectasis has been identified as a problem which was seen in our study as well ${ }^{12}$.

The commonest aetiology in this series, post infectious $(50 \%)$, is the commonest identified in many similar studies. A study done in India with 80 patients identified an underlying aetiology in 64\% of which the commonest was post infectious ${ }^{8}$. Postinfectious and immunodeficiency were the major aetiological causes in a cohort of 148 children with bronchiectasis studied in Guangzhou, China ${ }^{13}$. However, in the same series, a cause was not identified in $34 \%$. In our study population an aetiological cause was not identified in $25 \%$ of children. Clinical features between bronchiectasis patients with an identifiable aetiology and idiopathic were similar in our study as well as in the others in the published literature. Therefore the clinical features of bronchiectasis are not helpful in identifying the underlying cause. Systematic review of 12 studies with a total of 989 children with non-CF bronchiectasis has revealed that $64 \%$ of the subjects had an underlying disorder. Post infectious (17\%) had been the commonest. Persistent cough had been a symptom of $77 \%$ children in that review ${ }^{9}$.

Recurrent respiratory tract infections and the persistence of moist cough were the presenting symptom in the great majority of children in our group but only $22 \%$ had been referred electively for evaluation for chronic lung diseases. All the others were detected when admitted to a tertiary centre with an exacerbation of their respiratory symptoms. Despite having persistent lung signs and 
positive chest $\mathrm{X}$-ray changes, there had been a delay in diagnosing bronchiectasis, probably due to lack of knowledge of its existence as an important entity of poorly resolving respiratory conditions. Another possible factor could be the health seeking behaviour of the majority of patients where they desperately visit several medical practitioners on self-referral with poor symptom resolution. It should be emphasized that paediatric patients with poor symptom control with inhaler therapy be reevaluated by an experienced person as most of the bronchiectasis patients had been managed at outpatient level with inhalers as asthma.

The microorganisms that were found in our patients were Haemophilus influenzae type b, Moraxella and Pseudomonas, which was the common pattern that had been described in other series as well. Spirometry to assess lung functions was not used by most studies and 23 children in our group were able to perform a spirometry test where the commonest identified pattern was restrictive lung disease. Non-CF bronchiectasis is not an uncommon illness among Sri Lankan children. A high degree of suspicion is needed for early diagnosis, especially in children with complicated pneumonia. There is a need to create awareness about the importance of early diagnosis of bronchiectasis and the importance of correct postural drainage techniques and follow up as it would lead to less complications and better prognosis later in life.

\section{Conclusions}

An identifiable cause of non-cystic fibrosis bronchiectasis was found in $75 \%$ patients. The commonest cause identified was post infective $(50 \%)$

\section{References}

1. Kliegman RM, Stanton BMD, St. Gerne J, Schor NF, editors, Nelson Textbook of Paediatrics, 20 $0^{\text {th }}$ edition. Philadelphia: Elsevier; 2016.

2. Kapur N, Karadag B. Differences and similarities in non-cystic fibrosis bronchiectasis between developing and affluent countries Paediatric Respiratory Reviews 2011; 12(2); 91-6.

https://doi.org/10.1016/j.prrv.2010.10.010 PMid: 21458736

3. Fakhoury K, Kanu A. Management of bronchiectasis in children without cystic fibrosis. Available from:

https://www.uptodate.com/contents/manag ement-of-bronchiectasis-in-children- without-cystic-fibrosis (viewed $12^{\text {th }}$ September 2017)

4. Babayigit A, Olmez D, Uzuner N, Cakmakci H, Tuncel T, Karaman O. A neglected problem of developing countries: Non-cystic fibrosis bronchiectasis. Annals of Thoracic Medicine 2009; 4:21-4.

https://doi.org/10.4103/1817-1737.44781

PMid: 19561918 PMCid: PMC2700478

5. Chang AB. Bronchiectasis: so much yet to learn and to do. Paediatric Respiratory Reviews 2011; 12:89-90.

https://doi.org/10.1016/j.prrv.2011.01.001

PMid: 21458735

6. Chang AB, Masel JP, Boyce NC, Wheaton G, Torzillo PJ. Non-CF bronchiectasis: clinical and HRCT evaluation. Pediatric Pulmonology 2003; 35(6):477-83.

https://doi.org/10.1002/ppul.10289

PMid: 12746947

7. British Thoracic Society Guidelines, Management of non-CF bronchiectasis. Thorax 2010; 65: 34-37

8. Kumar A, Lodha R, Kumar P, Kabra SK. Non-cystic fibrosis bronchiectasis in children: clinical profile, aetiology and outcome. Indian Paediatrics 2015; 52(1):35-7.

https://doi.org/10.1007/s13312-015-05638

9. Brower KS, Del Vecchio MT, Aronoff SC. The aetiologies of non-CF bronchiectasis in childhood: a systematic review of 989 subjects. BMC Pediatrics 2014; 14:299.

https://doi.org/10.1186/s12887-014-0299$\mathrm{y}$

PMid: 25492164 PMCid: PMC4275950

10. Agarwal R, Chakrabarti A, Shah A, Gupta $\mathrm{D}$, Meis JF, Guleria $\mathrm{R}$, et al. ABPA complicating asthma ISHAM working group. Allergic bronchopulmonary aspergillosis: review of literature and proposal of new diagnostic and classification criteria. Clinical and Experimental Allergy 2013; 43:850-73. https://doi.org/10.1111/cea.12141

PMid: 23889240 
11. European Respiratory Society. Complicated pneumonia in children, Breath Journal 2013; 9(3):210-22.

12. Bouyahia O, Essadem L, Matoussi N, Gharsallah L, Fitouri Z, Mrad Mazigh S, et al. Aetiology and outcome of bronchiectasis in children: a study of 41 patients. Tunis Med. 2008; 86(11):996-9.

PMid: 19213492
13. Guan WJ, Gao YH, Xu G, Lin ZY, Tang Y, Li HM, et al. Aetiology of bronchiectasis in Guangzhou, southern China. Respirology 2015; 20(5):739-48 https://doi.org/10.1111/resp.12528 PMid: 25819403 